\title{
Letter to the editor: Specificity of Zika virus ELISA: interference with malaria
}

M Van Esbroeck ${ }^{1}$, K Meersman ${ }^{1}$, J Michiels ${ }^{2}$, KK Ariën ${ }^{2}$, D Van den Bossche ${ }^{1}$

1. National Reference Center for Arboviruses, Department of Clinical Sciences, Institute of Tropical Medicine, Antwerp, Belgium

2. Unit of Virology, Department of Biomedical Sciences, Institute of Tropical Medicine, Antwerp, Belgium

Correspondence: Marjan Van Esbroeck (mvesbroeck@itg.be)

Citation style for this article:
Van Esbroeck M, Meersman K, Michiels J, Ariën KK, Van den Bossche D. Letter to the editor: Specificity of Zika virus ELISA: interference with malaria. Euro Surveill. 2016;21(21): pii=30237. DOI: http://dx.doi.org/10.2807/1560-7917.ES.2016.21.21.30237

Article submitted on 19 May 2016 / accepted on 26 May 2016 / published on 26 May 2016

To the editor: We read the study by Huzly et al. [1] with interest and agree on the high specificity of the Euroimmun Zika virus (ZIKV) ELISA (Euroimmun, Lübeck, Germany).

We evaluated the specificity of this test on convalescent samples of 10 PCR-confirmed dengue patients $(\mathrm{n}=3$ DENV-1, $\mathrm{n}=4$ DENV-2, $\mathrm{n}=2$ DENV-3 and $\mathrm{n}=1$ DENV-4) with high IgM antibody ratios and a positive $(n=9)$ or negative $(n=1)$ result for IgG antibodies. We also tested the assay on 10 samples with high titres of neutralising antibodies against yellow fever virus and on five samples positive for rheumatoid factor. Except for one borderline result (ratio between 0.8 and 1.1) of ZIKV IgM in the convalescent sample from a patient infected with DENV-1 after a stay in Thailand, all results for ZIKV IgM and IgG were negative.

However, when we tested samples from malaria patients with a current infection (thick smear and PCR-positive) with Plasmodium falciparum $(\mathrm{n}=12), P$. falciparum/P. ovale $(\mathrm{n}=1), P$. vivax $(\mathrm{n}=3), P$. ovale $(\mathrm{n}=5)$ or $P$. malariae $(n=5)$, or a recently treated $P$. falciparum infection (microscopy-negative, PCR-positive) $(n=8)$, 14 of these 34 samples tested positive or borderline for ZIKV IgM, IgG or both. Positive or borderline results for both ZIKV IgM and IgG were registered in two of 13 samples from patients with a current infection with $P$. falciparum (including the patient with the mixed $P$. falciparum/P. ovale infection) and in one of eight samples from patients with a recently treated $P$. falciparum infection. Nine samples tested positive or borderline for ZIKV IgM only: four from patients with a current $P$. falciparum, two each from patients with a $P$. vivax and recently treated $P$. falciparum infection, and one from a patient with a $P$. malariae infection. Finally, one of 13 samples from patients with a current $P$. falciparum infection and one of five samples from patients with a $P$. ovale infection tested positive for ZIKV IgG only.
Virus neutralisation tests could not demonstrate a ZIKV infection in 11 of the 14 samples with positive or borderline results. Notably, we confirmed a recent ZIKV infection in one patient with a recently treated $P$. falciparum infection who had travelled in several African countries in the first half of 2015 and most recently in Cameroon (ZIKV IgM and IgG ratios in the ELISA were 2.61 and 7.50 respectively). The results from two patients with $P$. ovale (ZikV IgG ratio: 1.84 ) and $P$. vivax (ZIKV IgM ratio: 0.87 ) infection were not conclusive. The samples from patients with a current or recently treated $P$. falciparum infection with false positive ZIKV ELISA results showed ratios between 1.10 and 6.93 for ZIKV IgM and between 0.92 and 7.02 for IgG. One sample from a patient with a $P$. vivax infection tested borderline for ZIKV IgM. The ELISA ratio for ZIKV IgM in the sample from the patient with a $P$. malariae infection was 1.10 .

False positive results were not correlated with parasite densities.

Plasmodium is known for its ability to trigger polyclonal B-cell activation resulting in the production of antibodies that are not microorganism-specific [2], possibly leading to false positive results in serological assays. Samples from malaria patients should therefore be included in panels used to evaluate the specificity of assays, particularly those detecting antibodies against tropical diseases.

Although the risk for malaria in South and Central America is limited and false positive results in the ZIKV ELISA due to Plasmodium infections may not pose a large problem in that part of the world, species distribution modelling has shown environmental suitability for ZIKV in a large part of tropical and sub-tropical regions including Africa where malaria is endemic [3]. According to the information available for our patient with a confirmed infection, active transmission of ZIKV may occur in Africa. We therefore believe that users 
of the ZIKV ELISA should be aware of the possible interference.

\section{Conflict of interest}

None declared.

\section{Authors' contributions}

KM conceived the study, KM, DVDB and MVE analysed the data, JM and KA performed and analysed neutralisation tests, MVE and DVDB wrote the manuscript.

\section{References}

1. Huzly D, Hanselmann I, Schmidt-Chanasit J, Panning M. High specificity of a novel Zika virus ELISA in European patients after exposure to different flaviviruses.Euro Surveill. 2016;21(16):30203. DOI: 10.2807/1560-7917. ES.2016.21.16.30203 PMID: 27126052

2. Scholzen A, Sauerwein RW. How malaria modulates memory: activation and dysregulation of B cells in Plasmodium infection.Trends Parasitol. 2013;29(5):252-62. DOI: 10.1016/j. pt.2013.03.002 PMID: 23562778

3. Messina JP, Kraemer MU, Brady OJ, Pigott DM, Shearer FM, Weiss DJ, et al. Mapping global environmental suitability for Zika virus. Elife. 2016;5(5):e15272.PMID: 27090089

\section{License and copyright}

This is an open-access article distributed under the terms of the Creative Commons Attribution (CC BY 4.0) Licence. You may share and adapt the material, but must give appropriate credit to the source, provide a link to the licence, and indicate if changes were made.

This article is copyright of the authors, 2016. 